International Journal of Engineering \& Technology, $7(2.26)(2018) 31-34$
International Journal of Engineering \& Technology
SPC
Website $:$ www.sciencepubco.com/index.php/IJET
Research paper

\title{
Literature review on multimodal biometrics
}

\author{
S. Arunarani ${ }^{1 *}$, R. Gobinath ${ }^{2}$ \\ ${ }^{1}$ Research Scholar, Department of Computer Science, VISTAS, Pallavaram, Chennai \\ ${ }^{2}$ Assistant Professor, Department of Computer Science, Prince Shri Venkateshwara Arts and Science College, Chennai Department of \\ Computer Science, VISTAS, Pallavaram \\ *Corresponding author E-mail: sarunaarani@yahoo.co.in)
}

\begin{abstract}
As technological reformation is widen, biometric systems substitute knowledge based and token based recognition systems. Confidential data are accessed by the user after the user is recognized by biometric systems. Efforts have been made to acquire more suitable prototype for recognizing human as multimodal biometrics has more severe concern because of noise in the sample and malfunctioning sensing devices. This paper gives a dual study related to multimodal biometrics, including a literature review of the prior work in authentication and the proposed evaluation approaches. First, we classify few epitome studies considered in last decades to show how this problem has been solved until now. Second, the paper gives a introduction about basic principles of the associated evaluation approaches, and then provide an extended evaluation framework based on the enrollment selection and also statistically convincing measures for evaluating quality metrics.
\end{abstract}

Keywords: Multimodal Biometrics; Fusion; Fingerprint; Face; Ear Biometrics

\section{Introduction}

In this technological revolutionary world, each and every individual give importance to security. Humans feel insecure about their personal confidential belongings as the technology widen. Through internet the passwords and the bank accounts are ripped off. A demand for developing a system originated where physical or behavioral features of a human body act as a password. Hence a security system is developed that composed of multiple biometric traits as a password for the system. There are several distinctive measurable descriptors for recognizing an individual. There are mainly three types of descriptors viz.

1) Token based descriptors

2) Knowledge based descriptors

3) Biometrics based descriptors

The token based and Knowledge based systems can be deceived easily by the interloper. But biometric traits are unique for every individual so they cannot be easily cheated by an intruder. So, biometric based descriptors are extensively used because of their uniqueness and robustness.

\section{Multimodal biometrics}

To increase accuracy multimodal biometrics are used to restrict the number of applicant for identification. To improve recognition rate more than one physiological or behavioral characteristic are utilized for verification and

identification. Physiological characteristics that can be used as biometric traits are fingerprints, face, vein patterns, iris, retina, ear, gait, hand geometry, voice and behavioral characteristics that can be used as biometric traits are keystroke patterns and signature. A normal human have all the biometrics within him or her. So among the $\mathrm{N}$ biometric traits, any two or more traits can be chosen for a multimodal system to do the identification. Authors in [4] have defined seven criteria for selecting accurate biometric traits based on the need of the system.

The factors used to compare the several biometric traits are universality, uniqueness, Permanence, Measurability, Performance, Acceptability and Circumvention. Comparison of several biometric traits based upon these factors is given in table I.

In table I, G stands for good characteristic, B stands for bad characteristic and G/B stands for neither a good nor a bad characteristic for the criteria. From table I, it is clear that one needs to always have trade off in these characteristic so as to have a particular biometric. As can be seen from Table I, biometrics can be relied upon are face, fingerprint and ear. 


\begin{tabular}{|c|c|c|c|c|c|c|c|}
\hline $\begin{array}{l}\text { BIOMET- } \\
\text { RICS }\end{array}$ & $\begin{array}{l}\text { UNIVERSAL- } \\
\text { ITY }\end{array}$ & $\begin{array}{l}\text { UNIQUE- } \\
\text { NESS }\end{array}$ & $\begin{array}{l}\text { PERMA- } \\
\text { NENCE }\end{array}$ & $\begin{array}{l}\text { MEASURABIL- } \\
\text { ITY }\end{array}$ & $\begin{array}{l}\text { PERFOR- } \\
\text { MANCE }\end{array}$ & $\begin{array}{l}\text { ACCEPTABIL- } \\
\text { ITY }\end{array}$ & $\begin{array}{l}\text { CIRCUMVEN- } \\
\text { TION }\end{array}$ \\
\hline FACE & $\mathrm{G}$ & $\mathrm{G} / \mathrm{B}$ & B & $\mathrm{G}$ & $\mathrm{G} / \mathrm{B}$ & $\mathrm{G}$ & $\mathrm{G}$ \\
\hline $\begin{array}{l}\text { FINGER- } \\
\text { PRINT }\end{array}$ & B & $\mathrm{G}$ & $\mathrm{G}$ & B & G & B & B \\
\hline $\begin{array}{l}\text { HAND GE- } \\
\text { OMENTRY }\end{array}$ & B & B & B & G & B & B & B \\
\hline IRIS & G & $\mathrm{G}$ & $\mathrm{G}$ & B & G & G/B & G/B \\
\hline VOICE & B & G/B & $\mathrm{G} / \mathrm{B}$ & B & $\mathrm{G} / \mathrm{B}$ & $\mathrm{G}$ & $\mathrm{G}$ \\
\hline EAR & $\mathrm{G}$ & $\mathrm{G}$ & B & $\mathrm{G}$ & $\mathrm{G}$ & $\mathrm{G}$ & $\mathrm{G}$ \\
\hline SIGNATURE & $\mathrm{G} / \mathrm{B}$ & G/B & $\mathrm{G} / \mathrm{B}$ & $\mathrm{G} / \mathrm{B}$ & $\mathrm{G} / \mathrm{B}$ & B & B \\
\hline DNA & B & B & B & B & B & $\mathrm{G} / \mathrm{B}$ & G/B \\
\hline $\begin{array}{l}\text { KEY- } \\
\text { STROKE }\end{array}$ & $\mathrm{G} / \mathrm{B}$ & $\mathrm{G} / \mathrm{B}$ & $\mathrm{G} / \mathrm{B}$ & $\mathrm{G} / \mathrm{B}$ & $\mathrm{G} / \mathrm{B}$ & $\mathrm{G}$ & G \\
\hline
\end{tabular}

\section{1) Performance}

Any biometric system generates two types of scores in matching phase viz. a genuine score and an impostor score.

A genuine matching score is generated when two feature vectors corresponding to the same individual are compared, and an impostor matching score is generated when feature vectors from two different individuals are compared [8]. Matching stage generates either a distance score or a similarity score. For a biometric system, similarity score if calculated must be high for the genuine person and distance score must be low for genuine person and vice versa After the matching phase different threshold points are taken and depending upon the score values, different metrics are calculated so as to evaluate the performance of a biometric system.

The evaluation about the performance of a biometric system is done from metrics such as

FALSE ACCEPT RATE OR FALSE MATCH RATE: FAR OR FMR gives the percentage of invalid inputs which incorrectly matches with a non-matching template in the database.

FALSE REJECT RATE OR FALSE NON-MATCH RATE: FRR OR FNMR gives the percentage of valid inputs which are incorrectly rejected.

GENUINE ACCEPTANCE RATE: GAR is another metric for FRR used to measure performance of a system. GAR=1-FRR RECEIVER OPERATING CHARACTERISTIC (ROC):

ROC plot is a graphical presentation of the trade-off between the FAR and the FRR.

2) Fusion Methods

Multimodal biometric Fusion levels are classified into two main categories based on whether fusion done before matching or fusion done after matching.

Sensor level fusion and feature level fusion are fusion before matching and match score level fusion and decision level fusion are classified as fusion after matching.

sensor level fusion in sensor level fusion information are extracted and combined from each of source image which produce fused image taken for identification. fusion can take place at pixel, signal or at feature level but the multiple details got from various images must be compatible for fusion. same biometrics samples can be obtained from multiple sensors or different biometrics from multiple sensors or multiple instances from same sensor can be used for sensor level fusion.

3) Feature Level Fusion

In this level of fusion features from various sensors or from multiple samples or from multiple traits is combined to form a resultant vector. In multimodal biometrics features obtained from multiple traits must be compatible with one another. Feature level fusion is rarely used due to complexity in computation and because of larger dimensions in fused features.

4) Matchscore Level Fusion

In this level of fusion match scores from each trait is calculated and combined to give the resultant score.

5) Decision level

In this level, fusion is done only after the decision output from each and every biometric is available. Then, the decision from each biometric trait is combined to give the final result. Match score level fusion are used commonly because of its simplicity, less storage requirements easily generated and lesser computational complexity. Match score level fusion is categorized into two methods i.e. (a) rule based fusion and (b) classification based fusion as shown in figure 1 .

Match score level fusion bind scores from various unimodal biometric systems. Algorithms for match score level fusion for multimodal system associate rule based fusion and classification based fusion techniques. Methods such as sum rule, product rule, linear weighted sum rule (LWSR), min rule, max rule, fuzzy rules, majority voting rule, etc. follows rule based fusion. Methods such as Naïve Bayes Classifier, J48 Decision Trees and Support Vector Machines and K-means clustering, etc., follows classification based fusion.

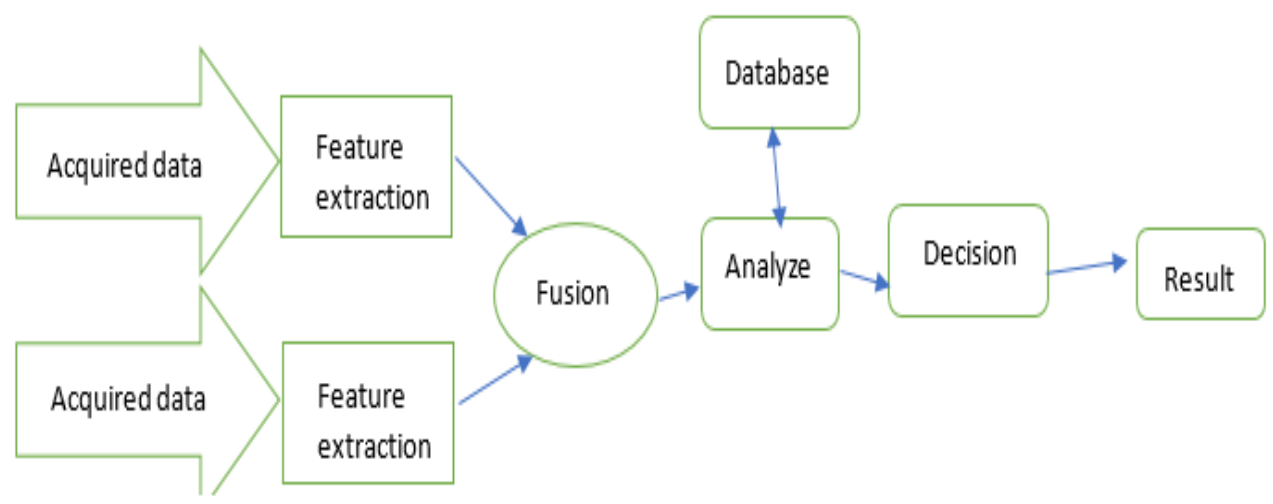

Fig. 1: Algorithms for Match Score Level Fusion. 




Fig. 2: Matching Score Level Fusion Techniques.

\section{Literature review}

Multimodal biometric systems overcome the problems that occur due to unimodal biometrics such as security problems and fault rates. Combining various biometric sources, multimodal biometrics offers high identification precision [12]-[18]. Several of the latest strategies have been offered beneath:

1) In He et al. (2010) [1], three biometric features were regarded in their study: fingerprint, face and finger vein. Support Vector Machines (SVM) -based score level fusion has been used. They join all the three modalities for verification. The fusion based on SVM classifier had attained higher accuracy of mean GAR of 0.999 and a mean FAR of $3 * 10-7$.

2) Pflug and Busch (2012) [2] have offered a review based on $2 \mathrm{D}$ and $3 \mathrm{D}$ ear biometrics, covering ear finding and ear identification systems. In 2D ear recognition strategies, holistic, local, hybrid and statistical techniques

3) Are used. They have created a smart surveillance system in which an ear detection and recognition strategies were presented. They also cleared many unsolved problems for ear recognition.

4) Huang et al. (2013) [3] have proposed a multimodal biometric system based on face and ear. This system use feature level fusion, which use Sparse Representation (SR). SR-based classification methods were used in multimodal classification phase. SR-based multimodal recognition techniques combined with Multimodal SRC with feature Weighting (MSRCW).

5) Islam et al. (2013) [4] the routine removal of Local 3D Features (L3DF) from ear and face. Hey used feature level fusion for 3D features and score level fusion for L3DF based matching. They offered algorithm based matching by means of a weighted sum rule. They have attained recognition and verification (at $0.001 \mathrm{FAR}$ ) rates of 99.0 and $99.4 \%$ on 3D ear and face.

Mohamad et.al [10] performed multimodal biometrics by combining the fingerprint and iris. They offered decision level fusion. A better performance and accuracy of $98 \%$ with FAR of $2 \%$, FRR of $2 \%$ and accuracy of $98 \%$ is attained by using fuzzy logic for fusion.

6) Gayatri Bokade et.al [11] combined face and palm print traits. They presented a feature level fusion system using a simple fusion algorithm. The GAR using palm images only is found to be $81.48 \%$. The GAR using face images is found to be $88.88 \%$. The fusion results indicate substantial increase in system to be $95 \%$, FAR of $0.5 \%$, FRR of $1.2 \%$

\section{Problem definition}

The distinctive feature of human for authentication is largely used in many fields such as forensic and criminal identification. Multi- modal biometric systems are extensively used in order to overcome the problems of unimodal sys

tems, such as noise, non-university, precision, spoof attacks etc.,. Very commonly used biometric features are face, fin

gerprint, ear, palm print and iris. Each biometric trait has its distinctive application, need, and benefits hence no one trait can be considered more superior than others. Among the available biometric modalities fingerprint, Face and ear have compatibility formation. Hence a multimodal biometrics system for person authentication by means of Fingerprint, Face and Ear is to be improved in order to overcome the issues of other multimodal biometric system.

1) Proposed Methodology

In the literature, recognition methods have been often offered with two modalities. However, the suggested method has important consideration due to the retention of actual distinctiveness. A multi-modal biometric recognition with fingerprint, face and ear modalities are applied now.

The proposed methodology includes of 4 phases such as

- Image Preprocessing,

- Feature extraction,

- Matching,

- Decision-making.

Image pre-processing

Image Preprocessing is proposed to improve the results. Input from fingerprint, face and ear image are pre-processed to remove the noise and shadow to have improved results. Cropping of the given fingerprint, face and ear images are done manually. Then the images are converted into the grayscale images. Filtering is applied to suppress noise and at the similar time, the signal is less deformed.

\section{2) Feature extraction}

In this phase, the grayscale images act as input to extract the shapes and texture characteristics of the fingerprint, face and ear images. PCA algorithm is used to extract the consistencies in the images.

3) Matching Module

A match score is calculated by comparing the extracted features with the templates in the database. This match score is used in the decision module to validate the identity.

4) Decision Making Module

Based on the match scores the decision-making module take decision on whether the user is an authorized user or an impostor.

\section{Conclusion}

Though different approaches are possible with suitable fusion levels are in practice, selection of appropriate modal for perfect multimodal biometrics which produces high performance. The different integration strategies that can be chosen to consolidate information were discussed here. The enhancement of security is possible by combining more than one biometric modalities. Ad- 
vanced security and high efficiency made the multimodal biometrics appropriate for all kinds of environments and system.

\section{References}

[1] He, M., S.J. Horng, P. Fan, R.S. Run, R.J. Chen, J.L. Lai, M.K Khan and K.O. Sentosa, 2010. Performance evaluation of score level fusion in multimodal biometric systems. Pattern Recogn, 43(5): 1789-1800.

[2] Pflug, A. and C. Busch, 2012. Ear biometrics: A survey of detection, feature extraction and recognition methods. IET Biometrics, 1(2): 114-129.

[3] Huang, Z., Y. Liu, C. Li, M. Yang and L. Chen, 2013. A robust face and ear based multimodal biometric system using sparse representation. Pattern Recogn., 46(8): 2156-2168.

[4] Islam, S.M.S., R. Davies, M. Bennamoun, R.A. Owens and A.S. Mian, 2013. Multibiometric human recognition using 3D ear and face features. Pattern Recogn., 46(3): 613-627.

[5] Aarohi Vora, Chirag Paunwala, Mita Paunwala, "Improved Weight Assignment Approach for Multimodal Fusion”, IEEE International Conference on Circuits, Systems, Communication and Information Technology Applications, CSCITA, pp.70- 74,April 2014.

[6] Aarohi Vora, Chirag Paunwala, Mita Paunwala, "Nonlinear SVM Fusion of Multimodal Biometric System", International Multi Conference on Innovations in Engineering and Technology, IMCIET 2014 under International Conference on Communication and Computing track, ICCC 2014, Elsevier, pp. 30-35, August 2014.

[7] Aarohi Vora, Chirag Paunwala, Mita Paunwala, "Statistical analysis of various kernel parameters on SVM based multimodal fusion,”Annual IEEE India Conference (INDICON), 2014, pp.1-5, Dec. 2014.

[8] A. Jain, K. Nandakumar, A. Ross, "Score Normalization in Multimodal Biometric Systems", Pattern Recognition, vol. 38, no.12, pp. 2270-2285, December 2005.

[9] Arun Ross, Anil Jain, "Information fusion in biometrics", Pattern Recognition Letters, Elsevier, vol. 24, no.13, pp. 2115- 2125, September 2003.

[10] Mohamad Abdolahi, Majid Mohamadi, Mehdi Jafari," Multimodal biometric system fusion using fingerprint and iris with fuzzy logic", International Journal of soft computing and engineering, Vol.2, Issue-6, 2013.

[11] Gayathri umakant bokade, ashok M.sapkal, "Feature level fusion of palm and face for secure recognition", International Journal of Computer and Electrical Engineering, Vol.4, No.2, 2012.

[12] Hema.C.R, Paulraj.M.P \& Ramkumar.S, "Classification of Eye Movements Using Electrooculography and Neural Networks", International Journal of Human Computer Interaction, Vol.5 (4), pp.51-63, 2014.

[13] Hema, C. R., Ramkumar, S., \& Paulraj, M. P. , "Idendifying Eye Movements using Neural Networks for Human Computer Interaction", International Journal of Computer Applications, 105(8), pp 18-26, 2014

[14] S.Ramkumar, K.SatheshKumar, G.Emayavaramban, "EOG Signa Classification Using Neural Network for Human Computer Interaction", International Journal of Computer Theory and Applications, Vol.9(24), pp.223-231, 2016

[15] Ramkumar, Dr.K.Satheshkumar and G.Emayavaramban" Nine States HCI using Electrooculogram and Neural Networks", IJET, Vol. 8(6), pp. 3056-3064, Jan 2017.

[16] S.Ramkumar, K.Sathesh Kumar G.Emayavaramban," A Feasibility Study on Eye Movements Using Electrooculogram Based HCI" IEEE- International Conference on Intelligent Sustainable Systems, pp.384-388, Dec-2017.

[17] G.Emayavaramban, S.Ramkumar, A.Amudha and K.Sathesh Kumar "Classification Of Hand Gestures Using FFNN And TDNN Networks", International Journal of Pure And Applied Mathematics, Vol.118 (8) Pp. 27-32, Jan 2018.

[18] S.Ramkumar, K.Sathesh Kumar, T.Dhiliphan Rajkumar, M.Ilayaraja, K.Shankar, "A review-classification of electrooculogram based human computer interfaces", Biomedical Research, 29 (6), Pp. 1078-1084, April 2018. 